\title{
Estratégias alimentares na larvicultura do peixe ornamental amazônico acará-severo (Heros severus) (Heckel, 1840)
}

\author{
[Feeding strategies on larval rearing of severum (Heros severus) (Heckel, 1840), \\ Amazonian ornamental fish] \\ D.A.V. Campelo ${ }^{1}$, I.C. Silva ${ }^{1}$, M.H.C. Marques ${ }^{1}$, B.J.C.F. Eiras ${ }^{1}$, \\ M.F. Brabo' ${ }^{1}$, L.B. de Moura' ${ }^{1}$, G.C. Veras $^{2}$ \\ ${ }^{1}$ Universidade Federal do Pará - Bragança, PA \\ ${ }^{2}$ Universidade Federal de Minas Gerais - Belo Horizonte, MG
}

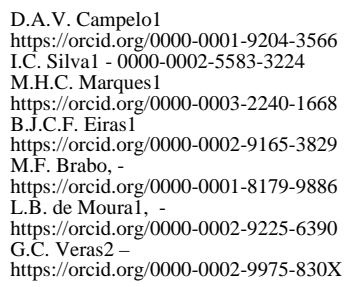

D.A.V. Campelo1 M.H.C. Marques 1 https://orcid.org/0000-0003-2240-1668 https://orcid.org/0000-0002-9165-3829

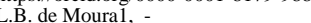
https://orcid.o https://orcid.org/0000-0002-9975-830X

\section{RESUMO}

Com o presente estudo, objetivou-se avaliar o tempo de fornecimento de náuplios de artêmia e o período de transição alimentar para pós-larvas de acará-severo (Heros severus). Foram utilizadas 450 pós-larvas de acará-severo, distribuídas em 45 aquários, em delineamento inteiramente ao acaso, em esquema fatorial $3 \times 5$, com três repetições. Foram avaliados três períodos de fornecimento de náuplios de artêmia: zero, cinco e 10 dias. Além disso, foram testados cinco períodos de transição alimentar: um, dois, três, quatro e cinco dias, quando os animais receberam alimentação conjunta de náuplios de artêmia e dieta farelada. Ao final do experimento, os peixes foram contados, pesados e fotografados, para avaliação do desempenho produtivo e das variáveis morfométricas. Para todos os parâmetros avaliados, não foi constatada interação entre o tempo de fornecimento de artêmia e o período de transição alimentar. Os animais alimentados por 10 dias com náuplios de artêmia apresentaram os melhores resultados de desempenho e as maiores variáveis morfométricas. Os peixes que receberam alimentação conjunta por três dias apresentaram desenvolvimento satisfatório. Dessa forma, recomendam-se 10 dias de fornecimento de náuplios de artêmia e três dias de transição alimentar, antes de se iniciar o fornecimento exclusivo de dieta inerte para pós-larvas de acará-severo.

Palavras-chave: náuplios de artêmia, transição alimentar, piscicultura ornamental, manejo alimentar

\begin{abstract}
The aim of this study was to evaluate the supply time of artemia and the period of food transition to postlarvae of severum (Heros severus). A total of 450 post-larvae of severum were distributed in 45 aquaria, in a completely randomized design, in a $3 \times 5$ factorial scheme, with three replicates. Three feeding periods with artemia nauplii were evaluated: zero, five, and ten days. In addition, five feeding transitions were tested: one, two, three, four and five days, when the animals received joint feeding of artemia nauplii and dry diet. At the end of the experiment the fish were counted, weighed and photographed, to evaluate the productive performance and the morphometric variables. For all parameters evaluated, no interaction between the time of artemia supply and the feeding transition period was observed. Animals fed for 10 days with artemia nauplii showed the best performance results and the highest morphometric variables. The fish that received joint feeding for three days presented satisfactory development. Thus, 10 days of artemia nauplii supply and three days of feeding transition is recommended before starting the exclusive supply of diet inert to post-larvae of severum.
\end{abstract}

Keywords: artemia nauplii, food transition, food management, ornamental fish farming

Recebido em 4 de abril de 2018

Aceito em 25 de janeiro de 2019

E-mail:danielvc@ufpa.br 


\section{INTRODUÇÃO}

$\mathrm{Na}$ piscicultura ornamental, o acará-severo (Heros severus), peixe da família dos ciclídeos, destaca-se pela boa adaptação ao cativeiro. Além disso, essa espécie é procurada no mercado devido à grande exuberância e coloração de seus exemplares. O acará-severo é originário dos rios Orinoco, Amazonas e Negro, e encontrado principalmente no Brasil, na Venezuela e na Colômbia (Kullander, 2003). Na natureza, alimenta-se de pequenos invertebrados e material vegetal; quando criados em cativeiro, aceitam facilmente qualquer tipo de dieta (Alishahi et al., 2014). É um peixe pacífico, mas pode apresentar territorialismo nos períodos de reprodução (Stawikowski e Werner, 1998). As fêmeas dessa espécie podem depositar entre 200 e 1000 ovos, apresentam cuidado parental e têm preferência por desovar em substratos como rochas ou galhos (Kullander, 2003).

A produção de juvenis de peixes de alta qualidade e em larga escala constitui fator-chave para o crescimento sustentável da indústria da aquicultura (Conceição et al., 2009). Na produção de peixes, a larvicultura pode ser considerada uma das fases mais críticas, principalmente devido à falta de conhecimentos básicos sobre as necessidades e os manejos nutricionais durante essa fase (Mohamed et al., 2012). Larvas de peixes altriciais apresentam escassa reserva de vitelo e não aproveitam, de maneira eficiente, o alimento inerte (Kolkovski, 2001), uma vez que não possuem sistema digestório completamente formado no momento em que iniciam a alimentação exógena, sendo dependentes de alimentos vivos (Cahu e Zambonino, 2001; Tesser et al., 2005).

A substituição muito precoce do alimento vivo pelo inerte pode levar à redução no crescimento e na sobrevivência das pós-larvas (Puello-Cruz et al., 2010; Jelkic et al., 2012). Dessa forma, torna-se importante a adoção de estratégias para substituir gradualmente o alimento vivo pelo alimento inerte, assim como determinar o período ideal para se realizar a supressão total do alimento vivo (Portella e Dabrowski, 2008). A lógica do emprego da alimentação conjunta é adaptar precocemente o peixe à captura, ingestão, digestão e absorção dos nutrientes do alimento inerte, evitando a redução das taxas de crescimento e o aumento das taxas de mortalidade que uma transição alimentar brusca pode gerar (Tesser e Portella, 2006; Jomori et al., 2008). Assim, com o presente trabalho, objetivase avaliar o tempo ideal de fornecimento de náuplios de artêmia e o melhor período de transição entre o alimento vivo e a dieta inerte, para pós-larvas de acará-severo (Heros severus).

\section{MATERIAL E MÉTODOS}

O experimento foi realizado no Laboratório de Piscicultura (Lapis), da Faculdade de Engenharia de Pesca, do Instituto de Estudos Costeiros, da Universidade Federal do Pará - Campus Bragança, e aprovado pelo Comitê de Ética no Uso de Animais da Universidade Federal do Pará, Ceua/UFPA (Processo nº 7656100517).

Foram utilizadas 450 pós-larvas de acará-severo, com sete dias após a eclosão, apresentando peso e comprimento padrão médios de $2,25 \pm 0,35 \mathrm{mg}$ e $4,89 \pm 0,17 \mathrm{~mm}$, respectivamente. As pós-larvas foram obtidas por meio da reprodução dos casais no Laboratório de Piscicultura. Após a absorção do saco vitelínico, os peixes foram distribuídos aleatoriamente em 45 aquários, com volume de 1L, na densidade de uma pós-larva para cada $100 \mathrm{~mL}$ de água. Os aquários possuíam aeração individual, realizada através de mangueira de $2 \mathrm{~mm}$ ligadas a um compressor radial. O laboratório foi mantido em fotoperíodo de 12 horas.

$\mathrm{O}$ experimento foi conduzido em delineamento inteiramente ao acaso, em esquema fatorial $3 \times 5$, com três repetições. Foram avaliados três períodos de fornecimento de náuplios de artêmia: zero, cinco e 10 dias. Além disso, foram testados cinco períodos de transição alimentar: um, dois, três, quatro e cinco dias, quando os animais receberam alimentação conjunta de náuplios de artêmia e dieta seca. Após cada período de transição alimentar, as pós-larvas passaram a receber apenas a dieta, até completarem 30 dias de alimentação.

A frequência alimentar foi de quatro vezes ao dia, nos horários de oito, 11, 14 e 17 horas. A quantidade de alimento vivo fornecida foi de 250 náuplios de artêmia/pós-larva/dia, conforme preconizado por Abe et al. (2016). No período de transição alimentar, a quantidade foi ajustada para 150 náuplios de artêmia/pós-larva/dia e a ração foi ofertada até a saciedade aparente dos 
animais. Após a alimentação das 11 e 17 horas, todas as unidades experimentais eram sifonadas, sendo trocados aproximadamente $50 \%$ do volume útil de cada aquário, para a eliminação das fezes e possíveis resíduos alimentares, garantindo-se, assim, a qualidade da água e o bem-estar das pós-larvas.

A dieta utilizada foi formulada para conter $42 \%$ de proteína bruta e $4200 \mathrm{kcal} / \mathrm{kg}$ de energia bruta (Tab. 1). Após a formulação, os macroingredientes foram moídos e misturados aos microingredientes nas respectivas proporções. Em seguida, as dietas foram umedecidas, com $25 \%$ de água a $50^{\circ} \mathrm{C}$, peletizadas em moedor elétrico (G.PANIZ, MCR-22, SP, Brasil), secas em estufa de ventilação forçada (QUIMIS, SP, Brasil) a $50^{\circ} \mathrm{C}$ e armazenadas em refrigerador, na temperatura de $10^{\circ} \mathrm{C}$. Antes de serem fornecidas aos peixes, as dietas foram trituradas para que o tamanho dos péletes se adequasse à abertura bucal das póslarvas.

Para o cultivo do alimento vivo, foram eclodidos diariamente $5 \mathrm{~g} / \mathrm{L}$ de cistos de artêmia em recipiente transparente com água salinizada na concentração de $35 \mathrm{~g} / \mathrm{L}$ de sal. Os recipientes foram mantidos sob iluminação artificial durante 24 horas, com o auxílio de lâmpadas fluorescentes (15W) e aeração adequada. Após a eclosão, os náuplios de artêmia foram retirados por sifonagem, lavados em água corrente para retirada da água salinizada e transferidos para um béquer de $200 \mathrm{~mL}$. Em seguida, foi efetuada a estimativa da densidade de náuplios, determinando-se o número de náuplios contidos em uma amostra de $0,5 \mathrm{~mL}$ do béquer. A contagem dos náuplios foi realizada com o auxílio de placa de Petri, sob estereomicroscópio com aumento de 40x. Depois de estimada a densidade de náuplios, foi calculado o volume do béquer a ser fornecido em cada unidade experimental.

Diariamente foram monitorados os parâmetros de $\mathrm{pH}$, temperatura $\left({ }^{\circ} \mathrm{C}\right)$ e oxigênio dissolvido $(\mathrm{mg} / \mathrm{L})$ da água dos aquários. A medição foi realizada por meio de aparelho multiparâmetro (YSI-556 MPS). Além disso, os níveis de amônia total $(\mathrm{mg} / \mathrm{L})$ da água foram mensurados a cada dois dias, por meio de Kit LabconTest (Indústria e Comércio de Alimentos Desidratados Alcon Ltda., Brasil). Os parâmetros de qualidade da água foram semelhantes entre os tratamentos. Os valores de temperatura, oxigênio dissolvido, $\mathrm{pH}$ e amônia total foram, respectivamente, $26,34 \pm 0,3^{\circ} \mathrm{C} ; \quad 6,95 \pm 0,7 \mathrm{mg} / \mathrm{L} ; \quad 7,14 \pm 0,3 \quad$ e $0,50 \pm 0,2 \mathrm{mg} / \mathrm{L}$.

Tabela 1. Valores da composição percentual e química da dieta fornecida às pós-larvas de acará-severo (Heros severus)

\begin{tabular}{ll}
\hline Ingredientes & $\mathrm{g} / \mathrm{kg}$ \\
\hline Farelo de soja & 200,0 \\
Farinha de peixe & 560,0 \\
Fubá de milho & 90,0 \\
Farelo de trigo & 70,0 \\
Óleo de soja & 56,0 \\
Fosfato bicálcico & 15,0 \\
Premix vitam/min ${ }^{1}$ & 5,0 \\
DL metionina & 3,8 \\
BHT $^{2}$ & 0,2 \\
Composição química $^{3}$ & \\
Proteína bruta (\%) & 42,0 \\
Extrato etéreo (\%) & 11,0 \\
Fibra bruta (\%) & 1,5 \\
Energia bruta (kcal/kg) & 4200,0 \\
\hline
\end{tabular}

${ }^{1}$ Níveis de garantia por quilograma do produto: vit. A, 1.200.000UI; vit. D3, 200.000UI; vit. E, $12.000 \mathrm{mg}$; vit. K3, 2.400mg; vit. B1, 4.800mg; vit. B2, 4.800mg; vit. B6, 4.000mg; vit. B12, 4.800mg; ác. fólico, $1.200 \mathrm{mg}$; pantotenato $\mathrm{Ca}, 12.000 \mathrm{mg}$; vit. C, $48.000 \mathrm{mg}$; biotina, 48mg; colina, $65.000 \mathrm{mg}$; niacina, $24.000 \mathrm{mg}$; ferro, $10.000 \mathrm{mg}$; cobre, $6.000 \mathrm{mg}$; manganês, $4.000 \mathrm{mg}$; zinco, $6.000 \mathrm{mg}$; iodo, $20 \mathrm{mg}$; cobalto, 2mg; selênio, $20 \mathrm{mg}$. ${ }^{2}$ Buti-hidroxitolueno (antioxidante). ${ }^{3}$ Realizado de acordo com AOAC (Official..., 2000).

Após término do experimento, os peixes foram contabilizados e pesados em balança analítica com precisão de $0,1 \mathrm{mg}$ (GEHAKA AG 200) para determinação dos parâmetros de peso final, ganho de peso (GP), GP = peso final - peso inicial, taxas de crescimento específico (TCE), $\mathrm{TCE}=((\ln$ peso final $-\ln$ peso inicial $) /$ número de dias) $* 100$, uniformidade do lote para peso (UP), UP $=$ (número de peixes com peso $\pm 20 \%$ da média)/número total de peixes por unidade experimental) *100 e taxa de sobrevivência (TS), $\mathrm{TS}=$ (número final de peixes/número inicial de peixes) $* 100$.

As pós-larvas também foram fotografadas com câmera digital FinePIX S2800HD, por meio de estereomicroscópio. As imagens foram analisadas pelo pacote de análise de imagens ImagePro-Plus ${ }^{\circledR}$, para determinação das 
seguintes variáveis morfométricas: comprimento padrão (CP), comprimento de cabeça (CC), comprimento de tronco (CTR), comprimento pós-anal (CPA), altura de cabeça (AC) e altura do corpo (ACO). As referidas medidas estão de acordo com o preconizado por Alvarado-Castillo (2010).

As análises estatísticas foram realizadas utilizando-se o software SAEG (Sistema..., 2007). Foi avaliada a normalidade e a homocedasticidade dos dados pelos testes de Lilliefors e Bartlett, respectivamente. Em seguida, foi realizada análise de variância (ANOVA) e, quando apresentada diferença $(\mathrm{P}<0,05)$, foi empregado o teste de Tukey a $5 \%$ de significância.

\section{RESULTADOS}

Para todos os parâmetros avaliados, não foi constatada interação entre o tempo de fornecimento de náuplios de artêmia e o período de transição alimentar. Em todos os tratamentos, as pós-larvas apresentaram taxas de sobrevivência e uniformidade do peso satisfatório, independentemente do tempo de fornecimento de artêmia e do período de transição alimentar (Tab. 2).

Os animais alimentados por 10 dias com náuplios de artêmia apresentaram os melhores resultados de peso final em relação aos animais alimentados por zero e cinco dias; essa mesma tendência foi observada para o ganho de peso e a taxa de crescimento específico. Em relação ao período de transição alimentar, os peixes que receberam alimentação conjunta por cinco, quatro e três dias apresentaram valores de peso final, ganho de peso e taxa de crescimento específico estatisticamente iguais (Tab. 2).

Tabela 2. Desempenho produtivo (média desvio-padrão) de pós-larvas de acará-severo (Heros severus) submetido a diferentes estratégias de alimentação

\begin{tabular}{|c|c|c|c|c|c|}
\hline \multirow{2}{*}{ Tratamento } & \multicolumn{5}{|c|}{ Desempenho produtivo } \\
\hline & PF (mg) & GP (mg) & TCE $(\%)$ & UP $(\%)$ & TS (\%) \\
\hline \multicolumn{6}{|l|}{ Período de fornecimento } \\
\hline 0 dias recebendo artêmia & $26,19 \pm 3,94 \mathrm{c}$ & $23,94 \pm 3,94 \mathrm{c}$ & $0,55 \pm 0,15 \mathrm{c}$ & $55,26 \pm 20,28$ & $96,67 \pm 5,33$ \\
\hline 5 dias recebendo artêmia & $40,35 \pm 3,60 b$ & $38,10 \pm 3,60 b$ & $0,99 \pm 0,09 b$ & $58,67 \pm 8,62$ & $100.00 \pm 0,0$ \\
\hline 10 dias recebendo artêmia & $55,37 \pm 8,35 \mathrm{a}$ & $53,12 \pm 8,35 \mathrm{a}$ & $1,30 \pm 0,15 \mathrm{a}$ & $65,70 \pm 14,10$ & $98,67 \pm 2,31$ \\
\hline \multicolumn{6}{|l|}{ Transição alimentar } \\
\hline Um dia & $36,68 \pm 10,62 b$ & $34,43 \pm 10,62 b$ & $0,84 \pm 0,32 b$ & $58,89 \pm 8,15$ & $97,78 \pm 9,95$ \\
\hline Dois dias & $37,26 \pm 7,75 b$ & $35,01 \pm 7,75 b$ & $0,89 \pm 0,21 b$ & $55,43 \pm 15,94$ & $96,67 \pm 5,19$ \\
\hline Três dias & $44,04 \pm 11,47 \mathrm{ab}$ & $41,79 \pm 11,47 \mathrm{ab}$ & $1,03 \pm 0,28 \mathrm{ab}$ & $62,22 \pm 19,75$ & $98,89 \pm 1,98$ \\
\hline Quatro dias & $46,14 \pm 10,54 a$ & $43,89 \pm 10,54 a$ & $1,09 \pm 0,22 \mathrm{a}$ & $56,67 \pm 14,81$ & $100,00 \pm 0,00$ \\
\hline Cinco dias & $46,84 \pm 12,87 a$ & $44,59 \pm 12,87 \mathrm{a}$ & $1,10 \pm 0,26 \mathrm{a}$ & $66,17 \pm 12,29$ & $98,89 \pm 1,98$ \\
\hline Período de fornecimento & $* * *$ & $* * *$ & $* * *$ & NS & NS \\
\hline Transição alimentar & $*$ & * & $* *$ & NS & NS \\
\hline Interação & NS & NS & NS & NS & NS \\
\hline
\end{tabular}

Em cada coluna, valores seguidos de mesma letra não diferem entre si pelo teste de Tukey a 5\% de probabilidade $(n=3)$.

PF - peso final; GP - ganho de peso; TCE - taxa de crescimento específico; UP - uniformidade do peso; TS - taxa de sobrevivência.

ANOVA: NS - não significativo ( $\mathrm{P}>0,05) ; * \mathrm{P}<0,05 ; * * \mathrm{P}<0,01 ; * * * \mathrm{P}<0,001$.

Da mesma forma que para o desempenho produtivo, não foi constatada interação entre o tempo de fornecimento de náuplios de artêmia e o período de transição alimentar para as variáveis 
morfométricas avaliadas. As pós-larvas que receberam artêmia por 10 dias apresentaram as maiores variáveis morfométricas. Com relação à transição alimentar, os peixes que passaram por quatro e cinco dias de alimentação conjunta apresentaram resultados estatisticamente iguais para comprimento padrão, comprimento de cabeça e comprimento pós-anal (Tab. 3).

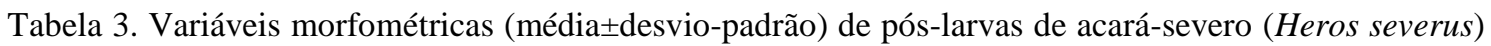
submetido a diferentes estratégias de alimentação

\begin{tabular}{|c|c|c|c|c|c|c|}
\hline \multirow{2}{*}{ Tratamento } & \multicolumn{6}{|c|}{ Medidas morfométricas } \\
\hline & $\mathrm{CP}(\mathrm{mm})$ & $\mathrm{CC}(\mathrm{mm})$ & CT (mm) & $\mathrm{CPA}(\mathrm{mm})$ & $\mathrm{AC}(\mathrm{mm})$ & $\mathrm{ACO}(\mathrm{mm})$ \\
\hline $\begin{array}{l}\text { Período de } \\
\text { fornecimento } \\
0 \text { dias } \\
\text { recebendo } \\
\text { artêmia }\end{array}$ & $8,61 \pm 0,06 \mathrm{c}$ & $3,09 \pm 0,02 \mathrm{c}$ & $5,47 \pm 0,53 \mathrm{c}$ & $3,28 \pm 0,04 \mathrm{c}$ & $2,56 \pm 0.03 c$ & $3,38 \pm 0,03 b$ \\
\hline $\begin{array}{l}5 \text { dias } \\
\text { recebendo } \\
\text { artêmia }\end{array}$ & $9,99 \pm 3,60 b$ & $3,70 \pm 0,03 b$ & $6,14 \pm 0,07 b$ & $4,04 \pm 0,04 b$ & $2,88 \pm 0,01 b$ & $4,41 \pm 0,56 b$ \\
\hline $\begin{array}{l}10 \text { dias } \\
\text { recebendo } \\
\text { artêmia } \\
\text { Transição } \\
\text { alimentar }\end{array}$ & $11,04 \pm 0,07 \mathrm{a}$ & $4,02 \pm 0,05 \mathrm{a}$ & $6,94 \pm 0,08 \mathrm{a}$ & $4,59 \pm 0,03 \mathrm{a}$ & $3,17 \pm 0,04 a$ & $4,87 \pm 0,74 \mathrm{a}$ \\
\hline Um dia & $9,56 \pm 0,04 \mathrm{c}$ & $3,47 \pm 0,04 \mathrm{c}$ & $5,72 \pm 0,09$ & $3,78 \pm 0,05 \mathrm{c}$ & $2,81 \pm 0,03$ & $3,81 \pm 3,81$ \\
\hline Dois dias & $9,57 \pm 0,07 \mathrm{c}$ & $3,49 \pm 0,02 b c$ & $5,93 \pm 0,13$ & $3,83 \pm 0,04 \mathrm{c}$ & $2,79 \pm 0,03$ & $3,82 \pm 0,05$ \\
\hline Três dias & $9,79 \pm 0,10 \mathrm{bc}$ & $3,59 \pm 0,04 b c$ & $6,10 \pm 0,08$ & $3,96 \pm 0,03 b c$ & $2,89 \pm 0,04$ & $4,50 \pm 0,72$ \\
\hline Quatro dias & $10,17 \pm 0,12 \mathrm{ab}$ & $3,78 \pm 0,04 \mathrm{ab}$ & $6,73 \pm 0,09$ & $4,06 \pm 0,04 \mathrm{ab}$ & $2,96 \pm 0,05$ & $4,13 \pm 0,14$ \\
\hline $\begin{array}{l}\text { Cinco dias } \\
\text { Período de } \\
\text { fornecimento }\end{array}$ & $\begin{array}{c}10,32 \pm 0,06 \mathrm{a} \\
* * *\end{array}$ & $\begin{array}{c}3,70 \pm 0,08 \mathrm{ab} \\
* * *\end{array}$ & $\begin{array}{l}6,44 \pm 0,02 \\
\quad * * *\end{array}$ & $\begin{array}{c}4,22 \pm 0,05 \mathrm{a} \\
* * *\end{array}$ & $\begin{array}{l}2,91 \pm 0,03 \\
* * *\end{array}$ & $\begin{array}{l}4,84 \pm 1,04 \\
* * *\end{array}$ \\
\hline $\begin{array}{l}\text { Transição } \\
\text { alimentar }\end{array}$ & $*$ & $*$ & NS & $*$ & NS & NS \\
\hline Interação & NS & NS & NS & NS & NS & NS \\
\hline
\end{tabular}

Em cada coluna, valores seguidos de mesma letra não diferem entre si pelo teste de Tukey a 5\% de probabilidade $(\mathrm{n}=$ 3).

$\mathrm{CP}$ - comprimento padrão; $\mathrm{CC}$ - comprimento de cabeça; $\mathrm{CT}$ - comprimento de tronco; $\mathrm{CPA}$ - comprimento pós-anal; AC - altura de cabeça; ACO - altura do corpo.

ANOVA: NS - não significativo $(\mathrm{P}>0,05) ; * \mathrm{P}<0,05 ; * * \mathrm{P}<0,01 ; * * * \mathrm{P}<0,001$.

\section{DISCUSSÃO}

No presente estudo, os animais alimentados por 10 dias com náuplios de artêmia apresentaram os melhores resultados de peso final, ganho de peso e taxa de crescimento específico. Para pós-larvas de acará-bandeira (Pterophyllum scalare), 20 dias de alimentação com artêmia é o período ideal para iniciar a transição alimentar (Pereira $e t$ $a l .$, 2016). Para não prejudicar a sobrevivência e o crescimento de pós-larvas de beta (Betta splendens), foram recomendados 15 dias de oferta de artêmia (Fosse et al., 2013). Azevedo et al. (2016) constataram que 12 dias de fornecimento de artêmia foram suficientes para pós-larvas de tricogaster (Trichogaster leeri), desde que a dieta seja suplementada com o simbiótico mananoligossacarídeo + B. subtilis.

A presença de enzimas digestivas nos organismos utilizados como alimento vivo pode proporcionar maior digestibilidade e melhor aproveitamento desse tipo de alimento pelos peixes, principalmente para as pós-larvas, que não possuem sistemas digestório e enzimático completamente desenvolvidos (Portella e Dabrowski, 2008). O alimento vivo também proporciona estímulos visuais e químicos, o que o torna mais atrativo para os peixes quando comparado às dietas artificiais (Tesser e Portella, 
2006). Dessa forma, para obtenção de taxas de sobrevivência e crescimento satisfatórias, a utilização do alimento vivo se torna extremamente importante no início da alimentação exógena de algumas espécies (Jomori et al., 2005). Por outro lado, segundo Pereira et al. (2016), quanto maior o tempo de fornecimento de náuplios de artêmia, menor a uniformidade do lote, devido principalmente ao estabelecimento de dominância entre as póslarvas. Para o acará-severo, os diferentes tempos de fornecimento de náuplios de artêmia e períodos de transição alimentar não afetaram a uniformidade do peso e a taxa de sobrevivência das pós-larvas.

As pós-larvas que passaram por um ou dois dias de transição alimentar apresentaram os piores valores de desempenho produtivo e variáveis morfométricas. $\mathrm{O}$ desenvolvimento reduzido desses animais pode ser explicado por diversos fatores, como o consumo inadequado de alimento, a redução na absorção de nutrientes devido à rápida taxa de passagem do alimento pelo sistema digestório dos peixes (Luz e Portella, 2005), a vantagem estabelecida pelos animais dominantes no momento da alimentação (Gonçalves Júnior et al., 2014), assim como a baixa atratividade do alimento inerte. A estratégia de transição alimentar é utilizada com o objetivo de melhorar a aceitação das pós-larvas ao alimento inerte. Esse procedimento melhora a digestão e a absorção dos nutrientes, uma vez que a presença do alimento vivo influencia diretamente na atratividade, na palatabilidade e na ingestão da dieta, estimulando o apetite e condicionando as pós-larvas ao alimento seco (Jomori et al., 2005; Tesser e Portella, 2006; Conceição et al., 2009).

Para pós-larvas de acará-severo, três dias de alimentação conjunta foi suficiente para que os animais apresentassem desenvolvimento semelhante aos que passaram por quatro e cinco dias de transição alimentar. Quanto menor o tempo de fornecimento do alimento vivo, menor os gastos com mão de obra, o que torna a criação das pós-larvas menos onerosa, uma vez que a produção e o fornecimento dos alimentos vivos podem representar quase $80 \%$ do custo total da larvicultura (Ruyet, 1993; Coraspe-Amaral et al., 2012). Da mesma forma que no presente estudo, pós-larvas de jundiá (Rhamdia quelen) que receberam alimentação conjunta por três dias apresentaram melhores resultados de desempenho quando comparadas àquelas que passaram por zero e sete dias de transição (Behr et al., 2000). Esses autores concluíram que o fornecimento de alimento vivo é essencial para o desenvolvimento adequado da espécie. $\mathrm{O}$ mesmo tempo de transição alimentar, três dias, também foi recomendado por Coraspe-Amaral et al. (2012) e Pereira et al. (2016), para pós-larvas de piaranha-do-pardo (Brycon sp.) e de acarábandeira, respectivamente.

Compreender os processos envolvidos no crescimento animal, como mudanças no tamanho e na forma do corpo, expresso por medidas ou índices morfométricos, é fundamental para o estabelecimento de novas técnicas de manejo (Reist, 1985; Santos et al., 2007). Segundo Nakatani (2001), as principais variáveis de crescimento são comprimento total, comprimento padrão, comprimento da cabeça e altura do corpo. Mudanças no manejo nutricional ocorridas durante as fases iniciais de criação podem refletir no crescimento alométrico dos peixes.

No presente trabalho, pós-lavas de acará-severo que receberam náuplios de artêmia por 10 dias apresentaram as maiores variáveis morfométricas. Com relação à transição alimentar, as pós-larvas apresentaram valores satisfatórios para a maioria das variáveis morfométricas a partir de três dias de alimentação conjunta, indicando que esse período é suficiente para realizar a troca do alimento vivo pelo alimento inerte. Pereira et al. (2016) observaram a mesma relação durante a larvicultura do acará-bandeira e recomendaram três dias de transição alimentar com base no crescimento e na sobrevivência dos animais. Os autores também afirmam que as pós-larvas de acará-bandeira alimentadas com alimento vivo até $10^{\circ}$ dia de vida, provavelmente, já apresentavam sistema digestório funcional, o que resultou em melhor aproveitamento do alimento inerte após a substituição do alimento vivo.

Manejo alimentar associando dietas secas a alimentos vivos proporcionam altos índices de crescimento e sobrevivência para diferentes espécies de peixes, principalmente em seus estágios iniciais de desenvolvimento (Furuya et al., 1999). Os resultados do presente estudo corroboram essa afirmação, tendo as pós-larvas 
de acará-severo alimentadas por um período de 10 dias com alimento vivo, seguido de três dias de transição alimentar, apresentado desenvolvimento muito semelhante àquelas que passaram por um período de transição de quatro e cinco dias.

É importante destacar que, por serem uma espécie ornamental, os peixes de menor tamanho também apresentam valor de mercado (Fujimoto et al., 2014). Como não ocorreram problemas com a uniformidade do peso e a taxa de sobrevivência, a realização de uma análise econômica pode auxiliar na escolha do melhor manejo a ser utilizado. Entretanto, para melhores índices de desempenho produtivo e variáveis morfométricas, pós-larvas de acará-severo devem ser alimentadas por 10 dias com náuplios de artêmia, seguidos de três dias de transição alimentar.

\section{CONCLUSÃO}

Os resultados obtidos no presente estudo permitem a implantação de protocolo mais eficaz de manejo alimentar para pós-larvas de acarásevero. O fornecimento de alimento vivo é fundamental durante a alimentação inicial dessa espécie e as pós-larvas apresentaram melhores resultados quando alimentadas por mais tempo com náuplios de artêmia. Recomendam-se 10 dias de fornecimento de náuplios de artêmia seguidos de três dias de transição alimentar, período em que os peixes devem receber alimentação conjunta de náuplios de artêmia e dieta inerte. Esse manejo alimentar é indicado para as pós-larvas de acará-severo estarem aptas a consumir unicamente o alimento inerte, sem prejuízos ao desenvolvimento dos peixes.

\section{REFERÊNCIAS}

ABE, H.A.; DIAS, A.R.; REIS, R.G.A. et al. Manejo alimentar e densidade de estocagem na larvicultura do peixe ornamental amazônico Heros severus. Bol. Inst. Pesca, v.42, p.514-522, 2016.
ALISHAHI, M.; KARAMIFAR, M.; MESBAH, M.; ZAREI, M. Hemato-immunological responses of Heros severus fed diets supplemented with different levels of Dunaliella salina. Fish. Physiol. Biochem., v.40, p.57-65, 2014.

ALVARADO-CASTILLO, J.D. Substituição precoce do alimento vivo por alimento inerte na larvicultura de acará bandeira (Pterophyllum scalare). 2010. 57f. Dissertação (Mestrado em Aquicultura) - Universidade Estadual Paulista, Jaboticabal, SP.

AZEVEDO, R.V.; FOSSE FILHO, J.C.; PEREIRA, S.L. et al. Prebiótico, probiótico e simbiótico para larvas de Trichogaster leeri (Bleeker, 1852, Perciformes, Osphronemidae). Arq. Bras. Med. Vet. Zootec., v.25, p.795-804, 2016.

BEHR, E.R.; TRONCO, A.P.; NETO, J.R. Ação do tempo e da forma de suplementação alimentar com Artêmia franciscana sobre a sobrevivência e o crescimento de larvas de jundiá. Cienc. Rural, v.30, p.503-507, 2000.

CAHU, C.; ZAMBONINO-INFANTE, J.L. Substitution of live food by formulated diets in marine fish larvae. Aquaculture, v.200, p.161180, 2001.

CONCEIÇÃO, L.E.C.; ARAGÃO, C.; RICHARD, N. et al. Avanços recentes em nutrição de larvas de peixes. Rev. Bras. Zootec., v.38, p.26-35, 2009.

CORASPE-AMARAL, M.V.; PEDREIRA, M.M.; COSTA, D.C. et al. Time of feed transition and inclusion levels of exogenous protease in rations for piabanha-do-Pardo (Brycon sp.). Acta Sci. Anim. Sci., v.34, p.347353, 2012.

FOSSE, P.J.; MATTOS, D.C.; CARDOSO, L.D. et al. Estratégia de co-alimentação na sobrevivência e no crescimento de larvas de Betta splendens durante a transição alimentar. Arq. Bras. Med. Vet. Zootec., v.65, p.1801-1807, 2013.

FUJIMOTO, R.Y.; SANTOS, R.F.B.; MARIA, A.N. Densidade de estocagem e manejo alimentar na criação de Acarás-bandeira. Aracajú: Embrapa, 2014. p.3-10. (Comunicado Técnico, n.145). 
FURUYA, V.R.B.; HAYASHI, C.; FURUYA, W.M. et al. Influência de plâncton, dieta artificial e sua combinação sobre o crescimento e sobrevivência de larvas de curimbatá (Prochilodus lineatus). Acta Sci. Anim. Sci., v.21, p.699-703, 1999.

GONÇALVES JUNIOR, P.L.; MENDONÇA, P.P.; PEREIRA, S.L. et al. Densidade de estocagem durante a larvicultura do kinguio. Bol. Inst. Pesca, v.40, p.597-604, 2014.

JELKIC, D.; OPACAK, A.; STEVIC, I. et al. Rearing carp larvae (Cyprinus carpio) in closed recirculatory system. Croatian J. Fish., v.70, p.917, 2012.

JOMORI, R.K.; CARNEIRO, D.J.; GERALDOMARTINS, M.I.E.; PORTELLA, M.C. Economic evaluation of Piaractus mesopotamicus juvenile production in different rearing systems. Aquaculture, v.243, p.175-183, 2005.

JOMORI, R.K.; CARNEIRO, D.J.; GERALDOMARTINS, M.I.E.; PORTELLA, M.C. Stable carbon $(\delta 13 \mathrm{C})$ and nitrogen $(\delta 15 \mathrm{~N})$ isotopes as natural indicators of live and dry food in Piaractus mesopotamicus (Holmberg, 1887) larval tissue. Aquacult. Res., v.39, p.370-381, 2008.

KOLKOVSKI, S. Digestive enzimes in fish larvae and juveniles-implications and applications to formulated diets. Aquaculture, v.200, p.181-201, 2001

KULLANDER, S.O. Family Cichlidae (Cichlids). In: REIS, R.E.; KULLANDER, S.O.; FERRARIS, C.J.J. (Eds.). Check list of the freshwater fishes of South and Central America. Porto Alegre: Edipucrs, 2003. p.605-654.

LUZ, R.K.; PORTELLA, M.C. Frequência alimentar na larvicultura do trairão (Hoplias lacerdae). Rev. Bras. Zootec., v.34, p.1442-1448, 2005.

MOHAMED, A.A.; EL-GALIL, A.; ABOELHADID, S.M. Trials for the control of trichodinosis and gyrodactylosis in hatchery reared Oreochromis niloticus fries by using garlic. Vet. Parasitol., v.185, p.57-63, 2012.

NAKATANI, H.K. (Ed.). Ovos e larvas de peixes de água doce: desenvolvimento e manual de identificação. Maringá: Eletrobrás, 2001. $378 \mathrm{p}$.
OFFICIAL methods of analysis. 17.ed. Gaithersburg: AOAC International, 2000. v.2.

PEREIRA, S.L.; GONÇALVES JUNIOR, L.P.; AZEVEDO, R.V. et al. Diferentes estratégias alimentares na larvicultura do acará-bandeira (Peterolophyllum scalare, Cichlidae). Acta Amaz., v.46, p.91-98, 2016.

PORTELLA, M.C.; DABROWSKI, K. Diets, physiology, biochemistry and digestive tract development of freshwater fish larvae. In: CYRINO, J.E.P.; BUREAU, D.; KAPOOR, B.G. (Eds.). Feeding and digestive functions in fishes. Londres: Science Publishers, 2008. p.227-279.

PUELLO-CRUZ, A.; VELASCO-BLANCO, G.; MARTÍNEZ-RODRÍGUEZ, I.E. et al. Growth and survival of siamese fighting fish, Betta Splendens, larvae at low salinity and with different diets. J. World Aquacult. Soc., v.41, p.823-828, 2010.

REIST, J.D. An empirical evaluation of several univariate methods that adjust for size variation in morphometric data. Can. J. Zool., v.63, p.1429-1439, 1985.

RUYET, J.P.L. Marine fish larvae feeding: formulated diets or live prey. J. World Aquacult. Soc., v.24, p.211-224, 1993.

SANTOS, V.B.; FREITAS, R.T.F.; LOGATO, P.V.R. et al. Rendimento do processamento de linhagens de tilápias (Oreochromis niloticus) em função do peso corporal. Ciênc. Agrotec., v.31, p.554-562, 2007.

SISTEMA para análises estatísticas-SAEG. Versão 9.1. Viçosa: UFV / Fundação Arthur Bernardes, 2007. 142p

STAWIKOWSKI, R.; WERNER, U. (Eds.). Die Buntbarsche Amerikas, band 1. Stuttgart, Germany: Verlag Eugen Ulmer, 1998. 50p.

TESSER, M.B.; CARNEIRO D.J.; PORTELLA, M.C. Co-feeding of pacu (Piaractus mesopotamicus, Holmberg, 1887) larvae with Artêmia nauplii and microencapsulated. J. Appl. Aquacult., v.17, p.47-59, 2005.

TESSER, M.B.; PORTELLA, M. Ingestão de ração e comportamento de larvas de pacu em resposta a estímulos químicos e visuais. Rev. Bras. Zootec., v.35, p.1887-1892, 2006. 